D01: 10.12957/demetra.2016.22493

\title{
Orden de Género e Insatisfacción Corporal: un análisis cualitativo
}

\section{Order of Gender and Body Dissatisfaction: a qualitative analysis}

\author{
Maria Teresa Valverde Martín \\ Marta Gutiérrez Sastrel \\ ${ }^{1}$ Universidad de Salamanca, Facultad de Ciencias \\ Sociales. Salamanca, España \\ Correspondencia / Correspondence \\ Teresa Valverde \\ E-mail: teresavalnet@hotmail.com
}

\section{Resumen}

Basándose en el estudio del cuerpo en relación a las normas socioculturales y al contexto histórico en que éste se sitúa, el artículo aborda, desde una perspectiva de género, la vivencia de las relaciones insatisfactorias con el cuerpo propio. La investigación utiliza metodología cualitativa para encontrar relatos íntimos sobre la corporeidad tensa. Se han realizado un total de 16 entrevistas semiestructuradas - 13 a mujeres y 3 a hombres - centradas en las experiencias de vida, en el peso de la imagen y en la vivencia personal con el cuerpo. Los resultados se articulan en torno a estos tres campos: en primer lugar, el impacto de la socialización y las relaciones familiares en la percepción de sí mismo a lo largo de todo el ciclo vital pero especialmente en la infancia y la adolescencia; en segundo lugar, la supeditación de la salud y la belleza a una imagen estereotipada de género; y, en tercer lugar, la desigual vivencia de la corporeidad tensa en hombres y mujeres, que se limita al aspecto físico en los primeros, mientras que para las segundas adquiere un tinte personal y emotivo angustioso. En relación a otros estudios, se confirma la pervivencia de una relación con el cuerpo diferencial entre hombres y mujeres.

Palabras clave: Imagen Corporal. Conducta Alimentaria. Identidad de Género.

\section{Abstract}

This article deals with the unsatisfactory experience with one's own body from a gender perspective. The research uses 
qualitative methodology to analyze personal accounts about the fraught corporeity. Sixteen semi-structured interviews have been made; they were focused on life experiences, self- image and the personal experience with body. The results are organized around three fields. Firstly, socialization and family relationships in perception of oneself; secondly, the subordination of health and beauty to a stereotypical image of gender; finally, the uneven experience of fraught corporeity for men and women, limited to the physical aspect for men, and full of distressing and emotional tinge for women. Compared with other studies, this research confirms the distinct relationship with the body between men and women.

Key words: Body Image. Feeding Behavior. Gender Identity.

\section{Introducción}

Las Ciencias Sociales han compartido con las Ciencias Naturales su preocupación por el cuerpo como objeto de estudio señalando, en este caso, la necesidad de abordar la corporeidad en relación a las normas sociales, los vínculos y el contexto sociohistórico en el que los cuerpos se ven sometidos.

Desde este ángulo, no se pueden obviar los trabajos que ven en el cuerpo una manifestación de la dominación y el control, pero también de la resistencia ${ }^{1}$ los que lo entienden como un espacio personal en el que se inscriben los valores y significados sociales; ${ }^{2}$ o los que encuentran en él un ejemplo de las crisis de la modernidad. ${ }^{3}$ Atendiendo a los análisis microsociólogicos el cuerpo se ha percibido como una representación de la cotidianeidad a partir de ciertos atributos corporales, ${ }^{4}$ o bien como eje de la relación con el mundo a través de las simbologías propias. ${ }^{5}$

En el estudio del cuerpo, el género, como la construcción arbitraria de lo biológico es, junto a la edad, la etnia o la clase social una de las principales categorías utilizadas. El género, como un artefacto cultural que modela al sujeto, ${ }^{6}$ confiere a las diferencias sexuales significado social y dirige la interpretación de las conductas, actividades y funciones de hombres y mujeres hacia el plano cultural.

Esta noción de género nos permite dar cuenta del modo en el que se configura un orden corporal generizado; un orden en el que la construcción del cuerpo masculino y femenino naturaliza el uso y disfrute androcéntrico del poder. A su vez, las representaciones sociales de dicha ordenación definen las expectativas y condiciones de vida de cada grupo sexual.

Los trabajos de Judith Butler ${ }^{7,8}$ niegan la corporeidad natural, ahondando en la construcción cultural de los cuerpos. Al negar la esencia natural del cuerpo, lo que encontramos son cuerpos 
culturalmente moldeados que portan las inscripciones narrativas de la historia y cuya materialidad corporal no es sino un efecto del poder. Esta concepción androcéntrica del poder se plasma en valoraciones corporales muy diferentes según se traten de hombres y mujeres. Según BustosRomero, ${ }^{9}$ la concepción del cuerpo y el atractivo físico en las mujeres cumple la función de ser para otros, ya sea para la procreación o como objeto de deseo de otras personas, mientras que en los hombres esta concepción del cuerpo y el atractivo físico se vincula con el propio poder masculino y el control.

El estudio de la corporeidad tensa, entendiendo por ello el análisis de las relaciones insatisfactorias con el cuerpo, pone en relación el ideal sobre los cuerpos, los deseos por cumplir la norma y los procesos de construcción y modelado corporal. La relación de estos tres factores no se presenta de manera lineal y armoniosa, sino tensa y plagada de regresiones y conflictos.

Un ámbito donde esta corporeidad tensa se percibe con nitidez es el relacionado con los trastornos de alimentación. Las aproximaciones biologicistas a esta cuestión insisten en el carácter patológico del comportamiento, pero, desde un punto de vista social, se señala la necesidad de observar esta cuestión en relación al contexto en el que el cuerpo es interpretado socialmente. ${ }^{10}$

La construcción social del género corre en paralelo a la preocupación por el físico de las mujeres. ${ }^{11}$ La obsesión moderna por la perfección corporal como un instrumento para el éxito, la aceptación social y la felicidad presenta demandas diferenciales para hombres y mujeres, si para los primeros mantiene el ideal de fortaleza asociada a un cuerpo musculoso a ellas les exige un concienzudo trabajo corporal en pos de la delgadez. ${ }^{12}$ La representación corporal de la felicidad se materializa a través de modelos esbeltas y delgadas y de hombres musculosos, depilados y escultóricos. ${ }^{13}$ La fijación por la delgadez, sin embargo, va más allá de los cánones de belleza natural constituyendo una prueba de obediencia femenina.$^{14} \mathrm{El}$ uso de estrategias normalizadoras vinculadas al poder, ${ }^{15}$ dificulta la individualización corporal e incide en la búsqueda generalizada de cuerpos dóciles, capaces de auto-control y autodisciplina, dispuestos a transformarse y mejorarse al servicio de las relaciones de dominio y subordinación. El miedo a la gordura de las mujeres es, en realidad, el miedo al poder de éstas, ${ }^{16}$ ya que supone una resistencia a las construcciones sociales dominantes.

A través de las normas y las rutinas habituales, el cuerpo se convierte en un locus de la práctica directa del control social. ${ }^{17}$ El cuerpo es controlado - entrenado y formado - a través de la ordenación del tiempo, el espacio y la vida cotidiana. El cuerpo es así un "cuerpo dócil”.

Wolf explica como a medida que las mujeres avanzan en la obtención de mayores libertades y derechos, mayor es el imperativo que exige su adherencia a la ideología de la belleza. La mujer opulenta, educada y liberada del primer mundo puede disfrutar de libertades que en otros tiempos fueron inaccesibles para ellas. Sin embargo, cuantos más obstáculos son superados mayor es el peso y la presión sobre su imagen. Parece que perdida la mística femenina de la domesticidad, 
el uso de la belleza femenina sustituye la función de control social desempeñada por la tradición utilizándose como un arma política contra el avance de las mujeres.

El culto a la belleza y la delgadez como modelo a seguir se dirige hacia la obtención de aceptación social, estatus, amor y reconocimiento profesional. ${ }^{18,9}$ Buscando la transformación corporal como premisa para la trasformación de sí mismo, los ideales de belleza han de materializarse en medidas y protocolos de actuación concretos. La cultura no sólo establece la norma de un cuerpo delgado, sino que provee de los protocolos necesarios para su obtención. ${ }^{19}$

Las presiones sobre los estereotipos de género con frecuencia ahondan en la imagen corporal negativa, ${ }^{20}$ una insatisfacción corporal de rechazo hacia el propio cuerpo que comienza en edades muy tempranas. En este campo son varios los estudios que analizan el papel que ejercen los medios de comunicación y la publicidad en la normalización del cuerpo y la belleza femeninas. ${ }^{21}$ En estos trabajos se destaca el fuerte vínculo establecido entre el éxito social y una apariencia física estereotipada. La necesidad de mantener el control del cuerpo en el proceso hacia el éxito, una concreción que representa el deseo de mantener el control general en sus vidas, constituye uno de los aspectos reveladores del proceso.

Este artículo aborda, desde una perspectiva cualitativa, el estudio de la relación que hombres y mujeres mantienen con sus cuerpos. El objetivo de esta investigación se dirige a conocer, desde una perspectiva de género, las vivencias personales sobre el cuerpo que relatan las personas que mantienen una relación insatisfactoria con el mismo.

\section{Metodología}

De cara a recoger la tensión diferencial que hombres y mujeres mantienen con su cuerpo se ha utilizado metodología cualitativa que permitiera adentrarse en la intimidad de los significados atribuidos. El universo del estudio son las personas con una relación tensa con su cuerpo. De ellas se ha buscado una narración centrada en las expectativas sobre el cuerpo ideal, las experiencias vividas y los comportamientos de autocontrol vinculados a la comida, la actividad física o los arreglos estéticos.

Esto hace necesario indagar en los relatos para entender el contexto de los actores sociales. La interiorización del sistema de valores y normas sobre "el ser", "el sentir" y "el hacer" está íntimamente relacionada con la vivencia de las propias experiencias y con el modo de percibir el mundo; así como también estará relacionado con las estrategias de afrontamiento y resolución

de tensiones. ${ }^{22}$ En este sentido, la narración del universo de sentido de los actores nos facilita comprender su vivencia de las corporeidades tensas. 
La técnica de la entrevista nos ha permitido obtener los relatos de vida de sus protagonistas. Teniendo en cuenta la consideración de Bertaux, ${ }^{23}$ hay relato de vida desde el momento en que un sujeto narra a otra persona, investigador o no, un episodio cualquiera de su experiencia vivida.

A la hora de seleccionar a los informantes (Tabla 1) se ha utilizado la red de contactos de la propia entrevistadora. En un principio se contactó con una asociación de familiares, pero no fue posible captar a ninguna participante. La opción de utilizar las Unidades Hospitalarias fue descartada para trabajar sobre un discurso "no institucionalizado". En el proceso de contactación se utilizó la técnica "bola de nieve", de modo que se pudieron obtener contactos a partir de las propias entrevistadas.

Tabla 1. Características de la muestra. Salamanca y Madrid (España).

\begin{tabular}{|c|c|c|c|c|c|}
\hline $\begin{array}{l}\text { Nombre } \\
\text { (ficticio) }\end{array}$ & Edad & $\begin{array}{l}\text { Criterio de } \\
\text { elección }\end{array}$ & $\begin{array}{l}\text { Inicio del } \\
\text { Proceso }\end{array}$ & $\begin{array}{l}\text { Intensidad } \\
\text { del Proceso }\end{array}$ & Tratamiento médico \\
\hline María & 27 años & $\begin{array}{l}\text { Trastorno } \\
\text { alimentario }\end{array}$ & Adolescencia & Superándolo & $\begin{array}{l}\text { Tratamiento en psiquiatría } \\
\text { infantil (sin ingreso) }\end{array}$ \\
\hline Ana & $\begin{array}{l}32 \\
\text { años }\end{array}$ & $\begin{array}{l}\text { Trastorno } \\
\text { alimentario }\end{array}$ & Adolescencia & Superándolo & $\begin{array}{l}\text { Tratamiento psiquiátrico } \\
\text { y psicológico (sin ingreso) }\end{array}$ \\
\hline Luisa & $\begin{array}{l}36 \\
\text { años }\end{array}$ & $\begin{array}{l}\text { Control del } \\
\text { cuerpo }\end{array}$ & $\begin{array}{l}\text { A partir de } \\
\text { los } 27 \text { años }\end{array}$ & Superándolo & Ninguno \\
\hline Marta & $\begin{array}{l}33 \\
\text { años }\end{array}$ & $\begin{array}{l}\text { Trastorno } \\
\text { alimentario }\end{array}$ & Adolescencia & Superándolo & Ninguno \\
\hline Susana & $\begin{array}{l}29 \\
\text { años }\end{array}$ & $\begin{array}{l}\text { Trastorno } \\
\text { alimentario }\end{array}$ & Adolescencia & Superándolo & Ninguno \\
\hline Laura & $\begin{array}{l}31 \\
\text { años }\end{array}$ & $\begin{array}{l}\text { Trastorno } \\
\text { alimentario }\end{array}$ & Adolescente & Superándolo & Ninguno \\
\hline Iván & 25 & $\begin{array}{l}\text { Control del } \\
\text { cuerpo }\end{array}$ & Actual & $\begin{array}{l}\text { Con } \\
\text { Intensidad } \\
\text { alta }\end{array}$ & Ninguno \\
\hline Estela & 30 & $\begin{array}{l}\text { Trastorno } \\
\text { alimentario }\end{array}$ & Adolescencia & Superándolo & Ninguno \\
\hline $\begin{array}{l}\text { Inés } \\
\text { (madre } \\
\text { de Alba) }\end{array}$ & $\begin{array}{l}\text { Edad de } \\
\text { Alba: } 20\end{array}$ & $\begin{array}{l}\text { Trastorno } \\
\text { alimentario } \\
\text { de Alba }\end{array}$ & Actual & $\begin{array}{l}\text { Con } \\
\text { intensidad } \\
\text { Muy alta }\end{array}$ & $\begin{array}{l}\text { Ingresada (Alba) en } \\
\text { Unidad de día Especifica } \\
\text { Hospitalaria. }\end{array}$ \\
\hline
\end{tabular}




\begin{tabular}{llllll}
\hline $\begin{array}{l}\text { Nombre } \\
\text { (ficticio) }\end{array}$ & Edad & $\begin{array}{l}\text { Criterio de } \\
\text { elección }\end{array}$ & $\begin{array}{l}\text { Inicio del } \\
\text { Proceso }\end{array}$ & $\begin{array}{l}\text { Intensidad } \\
\text { del Proceso }\end{array}$ & Tratamiento médico \\
\hline $\begin{array}{l}\text { Virginia } \\
\text { (madre } \\
\text { de Rocío) }\end{array}$ & $\begin{array}{l}\text { Edad de } \\
\text { Rocío } 29\end{array}$ & $\begin{array}{l}\text { Trastorno } \\
\text { alimentario } \\
\text { de Rocío }\end{array}$ & Adolescencia & Superándolo & $\begin{array}{l}\text { Tratamiento psiquiátrico } \\
\text { y tratamientos de día en }\end{array}$ \\
\hline Noemí & 38 & $\begin{array}{l}\text { Control del } \\
\text { cuerpo }\end{array}$ & Actual & $\begin{array}{l}\text { Con } \\
\text { Intensidad }\end{array}$ & Ninguno \\
\hline Araceli & 31 & $\begin{array}{l}\text { Control del } \\
\text { cuerpo }\end{array}$ & Actual & Superándolo \\
\hline Carmen & 35 & $\begin{array}{l}\text { Control del } \\
\text { cuerpo }\end{array}$ & Actual & $\begin{array}{l}\text { Intensidad } \\
\text { alta }\end{array}$ & Ninguno \\
\hline Manuel & 34 & $\begin{array}{l}\text { Control del } \\
\text { cuerpo }\end{array}$ & Actual & $\begin{array}{l}\text { Intensidad } \\
\text { alta }\end{array}$ & Ninguno \\
\hline Noelia & 39 & $\begin{array}{l}\text { Control del } \\
\text { cuerpo }\end{array}$ & Actual & $\begin{array}{l}\text { Intensidad } \\
\text { media }\end{array}$ & Ninguno \\
\hline Jesús & 31 & $\begin{array}{l}\text { Trastorno } \\
\text { alimentario }\end{array}$ & Actual & $\begin{array}{l}\text { Intensidad } \\
\text { alta }\end{array}$ & Ninguno \\
\hline
\end{tabular}

Las entrevistas han tenido lugar en Salamanca y en Madrid (España) y tienen una duración que oscila entre la hora y media y las tres horas. Las personas entrevistadas elegían el momento y el lugar de encuentro (se ofrecía un lugar tranquilo distinto de las cafeterías y lugares públicos, donde se pudiese hablar sin interferencias).

Todas las personas participaron voluntariamente y firmaron un consentimiento informado. Para salvaguardar la identidad de los participantes, los nombres que aparecen a lo largo del artículo son seudónimos.

Se han realizado un total de dieciséis entrevistas que se corresponden con distintos perfiles sobre la experiencia tensa respecto al cuerpo, en relación con el grado de superación de las mismas y con la intensidad de la problemática. También, se ha tenido en cuenta el concepto de saturación o punto de la investigación de campo en el que los datos comienzan a ser repetitivos y no se logran aprehensiones nuevas importantes.

Del total de las dieciséis entrevistas, trece de ellas han sido realizadas a mujeres, y tres a hombres, el mayor peso que recae del lado de las entrevistas a mujeres tiene que ver con el hecho 
de que la incidencia es mayor en las mujeres y se trataría de conocer mejor la influencia de la construcción del género en ello.

En las entrevistas se ha intentado construir una situación que se asemejase a una conversación, por lo que se han realizado en el comedor de una casa, delante de una taza de café u otra bebida y donde se ha podido hablar con tranquilidad, sin interrupciones, y en un ambiente relajado.

Se iniciaron las entrevistas con preguntas generales y abiertas que facilitaron la narración de las participantes. Todas las/los participantes comenzaron desde un principio hablando de sus perspectivas y experiencias. Se creó un clima que permitió a las entrevistadas/os expresar sentimientos, creencias, percepciones y no únicamente recuerdos o pensamientos.

En las entrevistas no sólo se ha indagado sobre los detalles de las experiencias de las entrevistadas/os sino que se ha indagado, también, sobre los significados que le atribuían a las mismas, para construir el sentido social de su conducta. Se trataron temas muy íntimos y con gran carga emocional, por lo que en algunos momentos las entrevistadas lloraron al narrar su relato

El guión de la entrevista se ha estructurado del modo siguiente:

Datos de filiación: edad, lugar de su nacimiento, ubicación de su residencia, entre otros.

Relaciones con su familia, especialmente con sus padres y hermanos.

Relato de su infancia momentos relevantes, tristes, alegres, recuerdos

Relato de su adolescencia: estudios, intereses/hobbies, relaciones, preocupaciones de esta etapa.

En cuanto al momento actual: relaciones con su familia, pareja, amigos, trabajo.

Cuestiones sobre sus valoraciones personales con preguntas como:

¿qué había sido lo más importante a lo largo de su vida.? ¿cuál era el valor a la salud, las relaciones de pareja, el trabajo, la imagen?.

Cuestiones sobre el nivel de exigencia personal con preguntas del tipo :¿qué estás dispuesta a hacer?, ¿te sacrificas mucho?, ¿te has sacrificado mucho?

Cuestiones sobre las rutinas del cuerpo, en el sentido del cuidado que le dedicaban y la presión que les pudiera suponer la imagen ante los demás.

Cuestiones sobre los problemas vividos: ¿qué dicen los demás? ¿Cuáles han sido las problemáticas especificas? ¿qué pensabas en ese momento? ¿Cuáles han sido las reacciones- contrareacciones? (de madre-hija, por ejemplo), ¿Han existido episodios importantes -ingresos o peleas-?.

Cuestiones sobre las justificaciones personales.

Relato de la situación actual con cuestiones sobre los conflictos resueltos y no resueltos, la valoración personal del proceso y los problemas presentes. 
Dentro de las mujeres entrevistadas dos de ellas eran madres de mujeres con construcciones tensas del cuerpo, en su caso, se les han realizado las cuestiones anteriores en relación a las vivencias de su hija y a las suyas propias.

Las entrevistas han sido grabadas y posteriormente transcritas para poder recoger con exactitud las experiencias y significaciones que la persona entrevistada realizaba de las mismas. Los verbatines que aparecen en el texto son citas literales (identificadas por seudónimo)

\section{Resultados y Discusión}

El tema de las construcciones sociales del cuerpo y la insatisfacción corporal, que se ha tratado, hace referencia a dinámicas en las cuales el lenguaje juega un rol central en la búsqueda de explicaciones y soluciones, puesto que, el discurso es un lugar donde los prejuicios, estereotipos, representaciones negativas, se re-producen y su análisis permite leer la realidad social.

Para describir las trayectorias corporales desde un punto de vista de género, nos centramos en la forma diferente en la que los hombres y mujeres entrevistadas construyen sus trayectorias corporales.

Los resultados se presentan atendiendo a tres aspectos: El impacto de la socialización y la familia en el ciclo vital; Los estereotipos de género, salud y belleza y, en tercer lugar, la corporeidad tensa en hombres y mujeres.

\section{El impacto de la socialización y la familia en el ciclo vital}

La construcción biográfica, es un plano lineal, continuo, en el que la infancia y adolescencia resultan vitales. La presencia de elementos biográficos en la entrevista se justifica en la medida en que el recorrido por la infancia, la adolescencia y el periodo adulto permiten encontrar la reconstrucción narrada del ciclo vital del sujeto en sus aspectos fundamentales.

La construcción corporal tensa se conforma a lo largo del desarrollo vital de la persona, durante el mismo los sujetos asumen distintas posibilidades de elección a partir de su relación con su entorno familiar, sus pares, su entorno laboral o sus relaciones de pareja. Estas interacciones que se producen a lo largo de las distintas etapas evolutivas conforman una trama social compleja que puede reconstruirse, ${ }^{10}$ a través de los relatos de los entrevistados, para analizar sus carreras corporales tensas.

En el entorno familiar se enseña desde la infancia sus propios parámetros estéticoscomportamentales, dentro de los cuales pueden estar incluidos el seguimiento de una apariencia delgada, el refuerzo de las conductas de dieta y el rechazo al sobrepeso o la obesidad. ${ }^{24}$ 
El énfasis sociocultural del atractivo que se realiza desde la familia suele llevarse a cabo, sobre todo en el caso de las niñas, en colaboración y correspondencia con las madres. ${ }^{25}$

Yo de pequeña quería ser modelo, yo, quería ser modelo, quería tener un cuerpo perfecto, como mi madre me decía que era delgada y alta, entonces esos patrones me han hecho mucho daño (Marta)

Autores como Ricciardelli y McCabe, ${ }^{26}$ afirman que la presión para perder peso ejercida por la madre es un factor predictivo principal, tanto, de la insatisfacción corporal sentida por hombres o mujeres, como, de la posibilidad de que unas u otros se involucren, en etapas posteriores como la adolescencia, en estrategias para modificar el cuerpo.

En la infancia, también, cobra gran relevancia la relación con los pares. Las entrevistadas/os asocian los momentos más felices de su infancia con los juegos de infancia y con sus amigos, tal y como nos dice uno de los hombres que ha participado en el estudio.

La infancia es un momento feliz, yo me lo pasé muy bien, muy bien tengo muchos recuerdos, [...] yo sueño muchas veces que estoy en el patio de mi colegio jugando al futbol con mis amigos [...] recuerdo muchas cosas con cariño y con un poco de nostalgia (Manuel).

Sin embargo, en la infancia se pueden experimentar dificultades crónicas en las relaciones entre pares, como hostilidad y aislamiento. El ser identificado con el cuerpo puede ser un elemento de hostilidad y se puede sufrir la estigmación social por apartarse del estereotipo corporal exitoso, tal y como nos indica una de las mujeres entrevistadas:

Yo durante el colegio había sido como..., lo habia pasado mal porque a mí me ponían el mote de mastodonte porque era una chica muy alta, grande, y yo sí es verdad que tenía un pelín de complejo (María).

El alejarse de la imagen corporal "bella" establecida por la cultura de la "estética corporal", e interiorizada en esta etapa, puede suponer la estigmación social. ${ }^{27}$

En la etapa adolescente se acentúa la necesidad de ajuste a los ideales corporales marcados socialmente al ser una etapa de cambios físicos y psicológicos significativos en los que la construcción de la propia imagen cobra una importancia singular. En este periodo aumenta, en general, el interés y la preocupación por la imagen corporal. Sin embargo los aspectos que les preocupan a hombres y mujeres son diferentes, como podemos comprobar en los relatos. Los hombres entrevistados hacen referencia a la estatura y la masa corporal.

De niño ha sido así, es que he comido un montón [...] estuve gordo hasta que llegué a 6o o 7o de primaria que empecé el estirón, ya sabes que cuando llegas a la adolescencia tu cuerpo cambia, y yo a parte de dar el 
estirón, que empecé a crecer, también empecé adelgazar [...] con trece años media casi 1.80, [...] ahi ya sí que me quedé, pues, más estilizado (Manuel).

A las mujeres, por el contrario, les preocupa ser demasiado gruesas o demasiado altas, como nos indica una de ellas.

Uff, Sí, porque yo veía a mis amigas que eran delgaditas, eran monas, y yo me veía como un autentico monstruo y ves las fotos mías y luego dice todo el mundo -no eras fea, pero lo que a mí me generó fue -quiero ser como mis amigas (María).

La magnitud de los cambios corporales que se producen en la pubertad originan un aumento en el interés y una preocupación por la imagen corporal. Existiendo diferencias de género con relación al foco de interés y grado de satisfacción con la imagen corporal. A los hombres adolescentes les interesan aspectos como la estatura y la masa muscular, que están relacionados con el poder físico, mientras que a las mujeres adolescentes les interesan aspectos como la delgadez. En el caso de los hombres, en el periodo de crecimiento aumentan los índices de masa corporal y estatura, lo que les proporciona satisfacción corporal. Sin embargo, en el caso de las mujeres adolescentes, se ven enfrentadas a un desarrollo físico sexual disarmónico y desproporcionado en sus inicios, que contradice el ideal corporal establecido socialmente, ${ }^{28}$ que prima la delgadez por lo que a medida que crecen se sienten menos satisfechas con su cuerpo. ${ }^{29}$

La imagen corporal consiste en el conocimiento y representación simbólica global del propio cuerpo, es la manera en como uno se ve e imagina a sí mismo. ${ }^{30}$ Esta visión de la imagen corporal puede ser satisfactoria o, por lo contrario, ser negativa y producir insatisfacción corporal, tal y como comenta una de las entrevistadas.

No me gustaba mi cuerpo, no me gustaba mi espalda, no me gustaba mi rodilla, no me gustaba la cara, no me gustaba mi barriga, es que no me gustaba mi cuerpo, entonces, claro, cuando no te gusta tu cuerpo pues lo pasas fatal (Marta).

Una imagen corporal negativa puede provocar malestar, máxime si tenemos en cuenta que las mujeres interiorizan, durante esta etapa adolescente, que su cuerpo es importante y que deberá tener unas características determinadas para tener valor. Compararse con personas o amigas que cumplen con esos parámetros de belleza puede provocar una distorsión de su propia imagen corporal y el deseo de adelgazar para acercase a ese modelo de cuerpo que asumen como perfecto, tal y como nos indica una de las entrevistadas: 
Mis amigas si que estaban muy delgadas, y tal, yo las veía más guapas, más monas y tal, puede que me influyera un poco (Susana).

Si el cuerpo causa gran insatisfacción se puede querer domesticar con una restricción o con un consumo excesivo de comida. El aumento de las actividades físicas y la introducción de cambios en la dieta en la adolescencia guardan relación con estos procesos. Tal y como cuenta una madre en relación a su hija.

Se hacía pesas con las botellas de agua de litro y medio o dos litros, las llenaba de agua o de arena, y se pasaba por la noche haciendo abdominales y pesas [...] comía menos, sólo quería comer ensalada y ensalada o jamón york o queso fresco [...] se lo comía pero venía a buscarlo andando desde Santa Marta al Carrefour, mira los kilómetros que te estoy diciendo (Virginia [madre de Rocío]).

Los valores, en relación con el cuerpo, que la sociedad establece de forma directa o indirecta en forma de ideales, en ocasiones, son demasiado elevados y si se aspira a ellos se requiere de sacrificios extremos para su consecución -relativos al control de los alimentos o a la realización de gran cantidad de ejercicio. Así, nos indica una de las participantes del estudio, hablando del periodo de su adolescencia:

Empezaba a tontear, pues eso, con pastillas de adelgazar, con hacer más ejercicio de la cuenta, [...] vomitar (María).

Esto ha supuesto para muchos de los participantes del estudio basar en la comida todos sus pensamientos y sus actos cotidianos, sintiéndose sumamente dependientes de esa idea, con lo cual el alimento ha sido el eje en torno al que giraba su vida y el mundo de sus relaciones.

Me metí en una dinámica en la que no quería salir de casa, no quería ver a mis amigas [...] yo a mis amigas como que las dejé de lado, para mí mis amigas dejaron como un poco de existir, yo me metí en casa (María).

La ingestión de determinados alimentos provoca, un sentimiento de culpa, especialmente en las mujeres, que tienden a relacionar el deseo de ingerir estos alimentos con estados de ánimo negativos como el aburrimiento o el estrés. ${ }^{31}$

Bueno, que me hubiera, habido veces, que me hubiese pegado, que yo decía,-pero, iqué me he comido un pastel! (Estela). 
Además, una pobre imagen corporal en la adolescencia puede ir asociada a una baja autoestima. $\mathrm{Si}$ la adolescente se siente mal con su cuerpo puede que se sienta poco adecuada como persona y no valore sus capacidades.

Era muy insegura, porque estaba hecha una mierda, no sabía que quería en la vida, o sea, me sentía fatal con mi cuerpo [...] yo no valoraba nada de lo que tenía (Marta).

Si no le gusta su cuerpo es difícil que le guste la persona que vive en él. Asimismo, es muy difícil que aprecie indistintamente cualidades de destreza o trabajo o incluso inteligencia, separadamente del aprecio por el propio cuerpo, las mujeres, especialmente, suelen subordinar muchas de estas cualidades al atractivo. ${ }^{32}$

Las entrevistadas que, con el paso del tiempo, han aprendido a aceptarse a sí mismas y han ganado en seguridad, son más flexibles consigo mismas, porque al tener una mayor autoestima no necesitan buscar continuamente la aprobación de los demás y perseguir, ni la perfección ni la perfección corporal.

Ahora, por ejemplo, digo voy hacer esto, o voy a pasarme en algo de la cuenta o tal, perdónalo [...] no pasa nada, disfrútate, lo que he ganado con la madurez, es que ya soy mucho más flexible (Marta).

Pero no todas las entrevistadas, que han padecido trastornos alimentarios en su adolescencia, han ganado en autoestima o han cesado en la búsqueda de la perfección.

Creo que me exijo, a veces, demasiado, porque es como que me siento, como que no puedo hacer muchas cosas, y las estoy haciendo, y aún haciéndolas [...] pero ya es como que me veo muchas veces como muy inferior, muy tal (Laura).

En este sentido, siguiendo a Moreno, la tensión corporal puede aumentar hasta tal intensidad, que el proceso acabe derivando en la administración de la propia vida por la terapia profesional, pero también puede revertirse el proceso, deteniendo los actos que propician las disposiciones restrictivas hasta llegar a deshacerlas. ${ }^{10}$

Estereotipos de género, salud y belleza

La imagen corporal se va construyendo de forma paralela al desarrollo evolutivo y cultural de Ia persona y no es sólo cognitiva, sino que, está impregnada de valoraciones subjetivas y determinada socialmente, razón por la cual no es ajena a la identidad de género, según Cash et al. ${ }^{30}$ 
Esto implica, siguiendo a De Beauvorir, ${ }^{33}$ una subordinación de lo femenino, en la escala de poder, al masculino, algo que se refleja en la presión corporal que se recibe. La mujer es la principal sometida a los mandatos estéticos de la sociedad, debido a que las construcciones de género han posibilitado que la mujer sea considerada como un objeto resignificado en función del punto de vista del otro. La mujer es instruida para llamar la atención del hombre, siendo este mismo el que ha construido y mantenido dichos cánones estéticos, según esta autora, con el fin de controlarla.

Entonces, era más eso, para darle a él, no para mí, yo estar delgada fue por él, fue para él, o sea, para gustarle a él, para decir, que no mire a otra chica, que no trate con otra chica, porque yo pensaba que si el día $x$ me dejaba yo, bueno, mi mundo se iba a derrumbar (Laura)

El sentirse influenciada por el criterio del otro puede crear en la mujer una incomodidad corporal no justificada en base a la estatura y el peso, tal y como nos indica una madre en relación a su hija:

Dice que le dijo un día (se refiere al primer novio que tuvo su hija con 14 años), -"ijolín, vaya culo que estás echando!". Nunca estuvo gorda, porque con 1.70 pesaba 62 kilos, ese es un cuerpo de 10, como digo yo, pero bueno, que nunca fue, y eso a ella la marcó, se lo he oído decir, siempre, a ella, siempre, siempre, siempre, que esas palabras le han machacado el cerebro, mejor que se las hubiera metido encima (Virginia [madre de Rocio]).

Respecto a la presión sentida por hombres y mujeres respecto a la imagen, que nos sirve para ahondar en la vivencia diferencial del cuerpo, podríamos decir, atendiendo al relato de las entrevistadas, que existe socialmente una diferenciación entre la gordura femenina y la masculina, presentando culturalmente mayor indulgencia la gordura de los hombres que la de las mujeres.

Es más la imagen nuestra, no es lo mismo ver a un chico gordo que a una chica gorda, según la sociedad, o sea, tú ves a un chico gordo y no te llama la atención, pero ves a una chica gorda y, a lo mejor somos nosotras mismas, eh, las que empezamos, - qué gorda está esa, no sé qué, pobrecita, a lo mejor dices, - pobrecita, que yo creo que es un poco la sociedad, que te marca esas...(Luisa).

La construcción de los cánones estéticos conlleva una definición del cuerpo femenino que se hace en la interacción yo-otro, pero además, siguiendo a Bourdieu, ${ }^{34}$ participa toda la estructura social, porque en el fondo de esa interacción subyacen los esquemas de percepción del cuerpo basados en relaciones de comparación, en el caso del cuerpo femenino, entre la gorda y la delgada, la vieja y la joven, la fea y la bonita.

Me fijo mucho en las chicas, en lo delgadas que están, cómo visten, cómo, y yo no me veo así, entonces, claro, yo digo, me gustaría verme así (Susana). 
Estos factores siempre están mediando entre la mujer y su cuerpo, ya que la imagen y las reacciones que su cuerpo produce en los demás, y la manera en que ella percibe esas reacciones, están estructuradas de acuerdo con esos esquemas indicados por Bourdieu. Las entrevistadas proyectan sobre su propio cuerpo los esquemas de percepción del cuerpo, basados en las relaciones de comparación, y se juzgan a ellas mismas sobre esas bases.

Yo no puedo pretender estar como (nombra a una amiga) como (nombra a otra) que pesa diez kilos, no puedo, es que no puedo tenerlo (Noemí).

Esto ocurre, según Bourdieu, porque las propiedades corporales ideales, que son aprehendidas por medio de los esquemas sociales de percepción, llevan a una evaluación que se aplica de acuerdo a la posición que ocupa cada propiedad en el espacio social, los factores dominadores, que se privilegian con frecuencia, y los dominados, que quieren ser evitados, como, por ejemplo: flaco/gordo o grande/pequeño.

Para ti la felicidad es cada vez estar más delgada, cada día estar más delgada, más delgada, era eso, y ponerte ropa cada vez más ajustada y más corta y más tal, era todo, era venga y ahora tengo que entrar en más pequeño, más pequeño, más pequeño (Laura).

En este sentido, la imagen corporal que la mujer tiene de sí misma está determinada socialmente y la exigencia de ciertas características estéticas de tamaño, indicadas por las entrevistadas, trata de ubicarlas simbólicamente en una escala aceptada por la mujer, que es el sujeto que percibe, y por el que es percibido.

Si tú te ves mal pues ves que los demás te están viendo mal también, si ya llegas a verte tú mal, dices, cómo me verán los demás si yo me veo mal, sabes, y sí, bueno, mi entorno más cercano, bueno, una persona que te encuentres, a lo mejor, no te dice nada, porque no, pero, a lo mejor, compañeras, o mis padres o mis hermanas o mi pareja te dicen -jo, es que estás engordando un montón (Luisa).

La preocupación del hombre por su apariencia física le pueda llevar, también, a un férreo control alimenticio y a unos hábitos extremos de ejercicio, tal y como relatan los hombres que han participado en el estudio, el origen de ello, sin embargo, nos indica uno de los entrevistados, se hallaría en el intento de seguir la moda, de cuidar de manera minuciosa la apariencia, con el fin de conseguir un cuerpo acorde a la tendencia de la metrosexualidad. La consecución de ese modelo de cuerpo sería para él el fin en sí mismo, no se trataría de un medio para obtener la aprobación de los "otros" como manifestaban las mujeres del estudio. 
Como mucho, porque yo soy de constitución delgada, y quiero ganar volumen [...] el gimnasio siempre lo primero, yo te soy sincero, me acuesto y me levanto pensando a ver como lo hago para no faltar al gimnasio [...] empecé porque era como una moda, todo el mundo iba al gimnasio, todo el mundo tenía cuerpos, que la moda sigue ahora, porque esto de la metrosexualidad, y tal, y por eso me apunté, como siempre me ha gustado la ropa, que si tal, pues esto algo más, me gusta depilarme, ahora no, porque tal, pero me gusta [...] Era como otro aliciente, la ropa, el peinado, pues venga, el cuerpo (Iván).

Aunque los hombres reciban, del mismo modo que las mujeres, presión social, para alcanzar los cánones de belleza impuestos por la sociedad: ser fuertes, musculosos, altos y guapos, no lo interiorizan del mismo modo, puesto que, no se trataría de "ser para el otro", como en el caso de la mujer, sino que alcanzar el cuerpo que se pretende es un fin en sí mismo.

Por otro lado, como la salud y la belleza han formado en las sociedades actuales un tándem inseparable, la presión por conseguir una determinada imagen va unida a la presión por conseguir un cuerpo sano. La manera considerada como efectiva para conseguir los objetivos de evitar el declive físico y mental es, a la vez, considerada una forma de lograr la belleza. Por lo que a través de unos hábitos alimentarios considerados saludables y del ejercicio se pretende conseguir ambas cosas, una buena salud y un cuerpo que se adapte a los cánones corporales establecidos. Sin embargo, a pesar de que salud y belleza parecen ir a la par, la obsesión por la perfección y la belleza, pueden potenciar hábitos alimentarios y conductas inadecuadas poniendo, en algunos casos, en riesgo la salud.

Dije,-si es que no llego a ningún lado, me voy a quemar el esófago, de hecho, pues, problemas de afonía, claro, porque los jugos gástricos, los dientes, muchisimos problemas en la boca, porque te come el esmalte, es que, pero, bueno, ya me di cuenta bastante tarde, o sea (Estela).

Las mujeres y hombres del estudio manifiestan en sus discursos buscar un cuerpo saludable, aunque reconocen que les preocupa principalmente que tenga unas determinadas características físicas. Y que para ello llevan a cabo conductas que no siempre son saludables, aunque paradójicamente éstas les llevan a conseguir el cuerpo que socialmente es considerado como un cuerpo saludable además de bello.

Yo lo que hago sé que me va bien para mi salud, pero yo lo hago, más que todo, por el físico, yo quiero tener un buen físico y sé que haciendo eso lo voy a tener, porque, por ejemplo, con las claras de huevo, pues yo como muchísimas claras de huevo, y sé que ahí, no hay daño porque el colesterol y todo lo malo está en la yema, ipero comer todos los días clara de huevo!, yo digo, esto no tiene que ser bueno (Ivan) 


\section{La corporeidad tensa en mujeres y hombres}

Las mujeres participantes en el estudio consideraban la delgadez corporal como el instrumento para alcanzar la felicidad. Esto sucede, siguiendo a Giddens, ${ }^{35}$ porque en la sociedad contemporánea de consumo, la disciplina del cuerpo mediante la dieta y el ejercicio se ha convertido en una de las claves para conseguir un cuerpo atractivo y deseable que proporcionará placer, felicidad y realización personal, ya que, la realización personal está sujeta al grado en que los cuerpos se ajustan a las normas contemporáneas de salud y de belleza.

Tal y como nos indica una de las entrevistadas, consideraba la delgadez corporal como el medio de conseguir la felicidad, y aunque comprobó que de ese modo no la alcanzaba, el engordar le sigue produciendo tristeza en el momento que realizan su relato.

$Y$ he de decir también que yo pensé que cuando estuviese delgada sería mucho más feliz y no, no. Cuando te quedas delgada, que yo pensé que iba a estar feliz y todo, es cuando dejé de salir [...] yo me amargaba [...] dejé de salir, dejé de salir, dejé de hacer cosas. [...] cuando he estado como he sido yo realmente es cuando he estado con las personas que yo he querido [...] pero el adelgazar o el engordar puede ser de lo que más me entristezca sin dudarlo. [...] que digas, estoy más triste porque he engordado, es que es muy triste, me produce tristeza, triste, de decir, jo, así (Susana).

En las normas contemporáneas de salud y belleza, el cuerpo humano se presenta ahogado por una figuración metafórica y comercial de la perfección y de la felicidad simbolizada a través de esbeltas y delgadas modelos de pasarela y de hombres musculosos, depilados y escultóricos. ${ }^{36} \mathrm{En}$ el caso de las mujeres el atributo físico más valorado y asociado a la belleza es la delgadez mientras que en el caso del hombre lo sería la fortaleza asociada a un cuerpo musculoso. ${ }^{37}$

Y las mujeres, especialmente, suelen subordinar las cualidades de éxito, belleza, elegancia, higiene y salud a esta delgadez. ${ }^{38}$ Por lo que consideran esta delgadez- asociada a la perfección física- como instrumento para conseguir el éxito, la felicidad e incluso salud.

Sin embargo, en el caso de los hombres, la consecución del cuerpo deseado es el fin en sí mismo como nos indica uno de los hombres entrevistados.

yo quiero tener un buen físico y sé que haciendo eso lo voy a tener[...] yo lo hago por verme bien realmente, si es que tampoco sé, sí imagen, a mi me compensa mirarme en el espejo y que lo que vea me gusta (Ivan). 
La perfección corporal que se busca, tal y como nos indica uno de los entrevistados, tiene componentes incompatibles entre sí.

A mi me gustaría tener un cuerpo fuerte de gimnasio y estar delgado, y eso, a veces, no es compatible, no es compatible estar fuerte y estar delgado, entonces, de ahi, estoy entre uno y otro, pero, sí, yo siempre desde que he tenido uso de razón he tenido abdominales y entonces yo los intento mantener ante todo, de verdad. (Jesús).

Por lo que para conseguir esa perfección corporal, tal y como nos relatan los participantes del estudio, tanto hombres como mujeres, la exigencia personal es máxima, y no se limitaba a restringir el alimento.

[...] voy comiendo un poquito, retirando otro poquito, voy comiendo otro poquito, retiro otro poquito, y me como, como la mitad de un plato [...]voy cuatro días al gimnasio, a lo mejor, el sábado me voy de caza, y el domingo de pesca, o algo así, casi todos los días de la semana práctico deporte (Jesús).

Eran las tres de la tarde, comía y me iba, a veces, he hecho hasta veinte kilómetros andando, me ponía una malla de estas que guardan el calor por dentro y me iba andar, o sea, y para mi todo eso era, comer, en pleno verano, a las tres de la tarde, con todo el sol, para mi era andar, y andar, hacer ejercicio, montar en la bici, todo era vomitar, comer poco y hacer ejercicio (María).

En el caso de las mujeres entrevistadas esta exigencia personal va acompañada de un componente de control muy fuerte.

[...] una vez que tenía que comer porque mi cuerpo dijo que tenía que comer ahi fue como, -ufff, y encima tengo que comer, pues entonces si tengo que comer tiene que salir, o sea, si ni siquiera puedo controlar eso [...] veinte minutos de reloj y bebía agua, en lo que se disolvía un poco de tal, en diez minutos vomitaba, es que era un control absolutamente de todo (Estela).

Este proceso es narrado por las mujeres que han participado en el estudio con una evidente angustia vital.

[...] yo siempre he dicho que voy a ser una mujer siempre obsesionada con mi cuerpo y me agobia muchísimo [...] físicamente, a lo mejor, me gustaría verme mucho mejor de lo que yo físicamente me veo, pero, a veces, también lo pienso, es que yo nunca me voy a ver bien, o sea, no es ya que yo quiera luchar por un físico de una manera, es que nunca voy a poderlo tener, porque nunca me lo voy a ver, aunque lo tenga (María). 
Para conseguir esta perfección corporal, tanto los hombres como las mujeres del estudio, relatan que la exigencia personal que les supone es máxima, y no está limitada a restringir el alimento. Pero en el caso de las mujeres entrevistadas esta exigencia personal va acompañada de un componente de control muy fuerte y este proceso es narrado por ellas con una evidente fuerte angustia vital.

\section{Conclusiones}

El objetivo de la investigación, planteado en la introducción de este articulo, ha sido conocer, desde una perspectiva de género, las vivencias personales sobre el cuerpo que relatan las personas que mantienen una relación insatisfactoria con el mismo.

Respecto a ello decir que, la idea de lo corporal como algo modificable, a través de la nutrición, el ejercicio y los cuidados, propicia la concepción del cuerpo como una creación del propio del individuo, como algo que puede ser construido para tratar de alcanzar unos cánones de belleza corporal que se asocian al éxito personal y social.

Cada momento histórico conlleva un conjunto de valores sociales y culturales que dan forma a un modelo corporal socialmente aceptado. Y ciertas construcciones tensas del cuerpo expresan la ansiedad y los problemas que causa el intento de adecuación a esos modelos corporales.

Los valores, en relación al cuerpo, que la sociedad establece de forma directa o indirecta en forma de ideales, en ocasiones, son demasiado elevados y si se aspira a ellos se requiere de sacrificios extremos para su consecución.

Las conductas dirigidas a la consecución del canon de belleza corporal socialmente aceptado son muy valorados, hasta que éstas son llevados a límites que adquieren la dimensión de trastorno corporal, y son expresión de desviación. Cuando esto sucede, pasan a ser tratados como enfermedad.

Los patrones estéticos corporales son reflejo de los valores culturales de cada momento histórico. La existencia de un ideal de belleza, establecido y compartido socialmente, supone, tal y como han expresado las entrevistadas/os que han participado en esta investigación, una presión importante, que se ha traducido, en algunos de los casos, en hábitos de alimentación y ejercicio inusitados.

La necesidad de ajuste a los ideales corporales marcados socialmente es mayor o menor dependiendo de la edad y del género. Respecto a la edad, en la etapa adolescente se acentúa esta necesidad, puesto que es una etapa de significativos cambios físicos y psicológicos en los que la construcción de la propia imagen cobra una importancia singular.

En las sociedades actuales, se ha impuesto la delgadez como canon de belleza y se ha extendido, sobre todo entre las mujeres, la preocupación por no sentirse incluidas dentro de los estándares de pesos y formas que la cultura imperante presenta como óptimos. Compararse con personas 
que cumplen con esos parámetros de belleza puede provocar una distorsión de su propia imagen corporal y el deseo de adelgazar para acercase a ese modelo de cuerpo que asumen como perfecto.

La identidad de género toma gran relevancia en las construcciones corporales, porque el autoconcepto de cuerpo que cada persona tiene se construye desde una perspectiva de género. La idea de belleza, no es ajena a los estereotipos de género. Y el canon de belleza está en la base de las construcciones del cuerpo, ya que, en estas construcciones se siguen las normas estéticas que han sido interiorizadas en el proceso de socialización.

En el proceso de socialización, los individuos pertenecientes a una sociedad o cultura aprenden e interiorizan un repertorio de normas, incluidas las normas y valores corporales. Los principales agentes socializadores son la familia y la escuela.

Siendo la familia el primer agente socializador, sobre todo en lo que respecta a las funciones más primarias y que hacen referencia a sentimientos, valores y actitudes, dentro de los cuales se encuentran los valores, sentimientos y actitudes corporales, así como, las conductas que van a guiar la consecución del modelo corporal transmitido.

La familia constituye el primer subsistema social donde observar y practicar roles, ofreciendo modelos, en los que están incluidos los modelos corporales, en los que confluyen elementos diferenciadores, y que moldean las relaciones sociales que se ejercen en ámbitos más amplios. Dentro de las categorías adscritas al rol femenino nos encontramos, entre otras, con las de "cuidado". Se trata de "ser para otros", algo que en el ámbito corporal cobra, del mismo modo, gran repercusión.

Las relaciones entre pares, también, son esenciales para la adaptación social, puesto que, ayudan a manejar nuevas habilidades sociales y a acceder a las normas sociales y procesos involucrados en las relaciones interpersonales. Sin embargo, se pueden experimentar dificultades crónicas en las relaciones entre pares, como hostilidad y aislamiento, relacionadas con el elemento corporal.

Por tanto, el canon de belleza corporal internalizado en las etapas tempranas del desarrollo forma parte de los criterios que la persona tiene como referencia a la hora de establecer si se adecua a los patrones de belleza socialmente impuestos.

Además, las mujeres sienten la presión estética de una manera más intensa y de forma diferente a los hombres, puesto que el deseo de ajustarse a los cánones de belleza corporal se sustenta, tal y como nos indican las entrevistadas, en la necesidad de agradar a los demás. Sin embargo, los hombres entrevistados, aluden a que intentan alcanzar los ideales corporales, establecidos socialmente, para sentirse bien consigo mismos.

El cuerpo femenino como cuerpo percibido y vivido como un ser para otro está determinado por aquello que le caracteriza particularmente, como la estatura o el peso, y condicionado por el criterio estético establecido socialmente y transmitido por los otros. Esto puede crear en la mujer una incomodidad corporal que no está justificada en base a la estatura y el peso. 
Sin embargo, en el caso de los hombres aunque reciban presión social para alcanzar los cánones de belleza impuestos por la sociedad, no lo interiorizan del mismo modo y los hombres entrevistados no experimentan la necesidad de buscar la perfección "para el otro". La motivación de cuidarse para los demás, también, puede existir, tal y como nos comentan los entrevistados, pero aparece unida a la necesidad de gustarse a sí mismos.

Por otro lado, la salud y la belleza han formado un tándem inseparable, la manera considerada como efectiva para conseguir los objetivos de evitar el declive físico y mental es, a la vez, considerada una forma de lograr la belleza.

Por lo que a través de unos hábitos alimentarios considerados saludables y del ejercicio se pretende conseguir ambas cosas, una buena salud y un cuerpo que se adapte a los cánones corporales establecidos. Sin embargo, a pesar de que salud y belleza parecen ir a la par, la obsesión por la perfección y la belleza, pueden potenciar hábitos alimentarios y conductas inadecuadas poniendo, en algunos casos, en riesgo la salud.

\section{Referencias}

1. Foucault M. Historia de la sexualidad. v. 1: la voluntad del saber. Madrid: Siglo XXI; 1976.

2. Bourdieu P. El sentido práctico. Madrid: Taurus; 1991.

3. Turner B. El cuerpo y la sociedad. Exploraciones en teoría social. México: Fondo de Cultura Económica; 1989.

4. Goffman E. The presentation of everyday life. New York: Anchor Books; 1959.

5. Le Breton D. La sociología del cuerpo. Buenos Aires: Nueva Visión; 2002.

6. Lupton D. Medicine as culture: illness, disease and the body in Western societies. London: Sage; 1994.

7. Butler J. El género en disputa. México D.F: Paidós; 2001.

8. Butler J. Cuerpos que importan. Sobre los límites materiales y discursivos del "sexo". Buenos Aires: Paidós; 2005.

9. Bustos-Romero O. Los medios y la construcción de género: factor de riesgo para trastornos alimentarios como anorexia y bulimia. Derecho a Comunicar 2011; 2:61-79.

10. Moreno JL. Moral corporal, trastornos alimentarios y clase social. Madrid: CIS; 2010.

11. Corti A. La estética de la imagen y la tiranía del éxito. Revista Electrónica de Psicología Política [Internet] 2005; 2(8). Disponível em: http://www.psicopol.unsl.edu.ar/abril05_nota4.htm

12. Cabrera G. El cuerpo femenino en la publicidad. Revista Icono 2010; 14:223-243

13. Falk P. The consuming body. London: Sage publications; 1994. 
14. Wolf N. The body myth: how images of beauty are used against women. New York: Morrow; 1991.

15. Foucault M. Vigilar y castigar. Madrid: Siglo XXI; 1984.

16. Bordo S. Unbearable weight. Feminism, western culture and the body. Berkeley-Los Angeles-Londres: University of California Press; 1993.

17. Macclansy J. Consuming culture. London: Chapmans Publishers; 1990.

18. Crispo FE, Guelar D. Anorexia y bulimia: lo que hay que saber. Barcelona: Gedisa; 2001.

19. Calado M. Liberarse de las apariencias. Madrid: Pirámide; 2011.

20. Walzer A. La belleza: de la metafísica al spot. Madrid: Facultad de Ciencias de la Información. Universidad Complutense de Madrid; 2003.

21. Mingote Adán C. La medicina psicosomática desde una perspectiva de género. AAVV: II Jornadas de Salud Mental y Género; 09-10 oct. 2000; Madrid: Instituto de la Mujer; 2000. p. 15-30. Serie Debate, 32.

22. Bertaux D. Los relatos de vida. Perspectiva etnosociológica. Barcelona: Bellaterra; 2005.

23. Behar R. La construcción cultural del cuerpo: el paradigma de los trastornos de la conducta alimentaria. Revista Chilena de Neuropsiquiatría 2010; 48(4):319-334.

24. Mc Phail Fanger E. Cuerpo y cultura. Razón y Palabra [Internet] 2002; (25). Disponível em: http:// www.razonypalabra.org.mx/anteriores/n25/emcphail.html

25. Ricciardelli L, McCabe M. Children's body image concerns and eating disturbance: a review of the literature. Clin. Psychol. Rev. 2001; 21(3):325-44.

26. Corti A. La estética de la imagen y la tiranía del éxito. Revista Electrónica de Psicología Política [Internet] abr. 2005; 2:(8).

27. Maganto C, Cruz S. La imagen corporal y los trastornos alimenticios: una cuestión de género. Cuadernos de Psiquiatría y Psicoterapia del Niño y del Adolescente 2000; 30:45-48.

28. La pubertad. In: Adrián Serrano JE, Rangel Gascó E. Aprendizaje y Desarrollo de la Personalidad (SAP001). 2012; 5-9. Disponível em: http://www3.uji.es/ betoret/Instruccion/Aprendizaje $\% 20 y \% 20$ DPersonalidad/Curso $\% 2012-13 /$ Apuntes $\% 20$ Tema $\% 201 \% 20 \mathrm{La} \% 20$ transicion $\% 20$ adolescente $\% 20$ y\%201a\%20educacion.pdf

29. Cash T, Pruzinsky T. Body images: development, deviance and changes. Nueva York: Guilford Press; 1990.

30. Weingarten HP, Elston D. Food cravings in a college population. Appetite 1991; 17:167-175.

31. Raich RM. Una perspectiva desde la psicología de la salud de la imagen corporal. Avances en Psicología Latinoamericana 2004, 22:15-27.

32. De Beauvoir S. El segundo sexo. Buenos Aires: Sudamericana; 1967

33. Bourdieu P. La distinción: criterio y bases sociales del gusto. Madrid: Taurus; 1988.

34. Giddens A. The constitution of society: outline of the theory of structuration. Cambridge: Polity; 1984. 
35. Falk P. The consuming body. London: Sage Publications; 1994.

36. Cabrera G. El cuerpo femenino en la publicidad. Revista Icono 2010; 14:223-243.

37. Raich RM. Una perspectiva desde la psicología de la salud de la imagen corporal. Avances en Psicología Latinoamericana 2004; 22:15-27.

Recebido: $15 / 4 / 2016$

Revisado: 03/8/2016

Aceito: 30/8/2016 